\title{
Octobre 2005
}
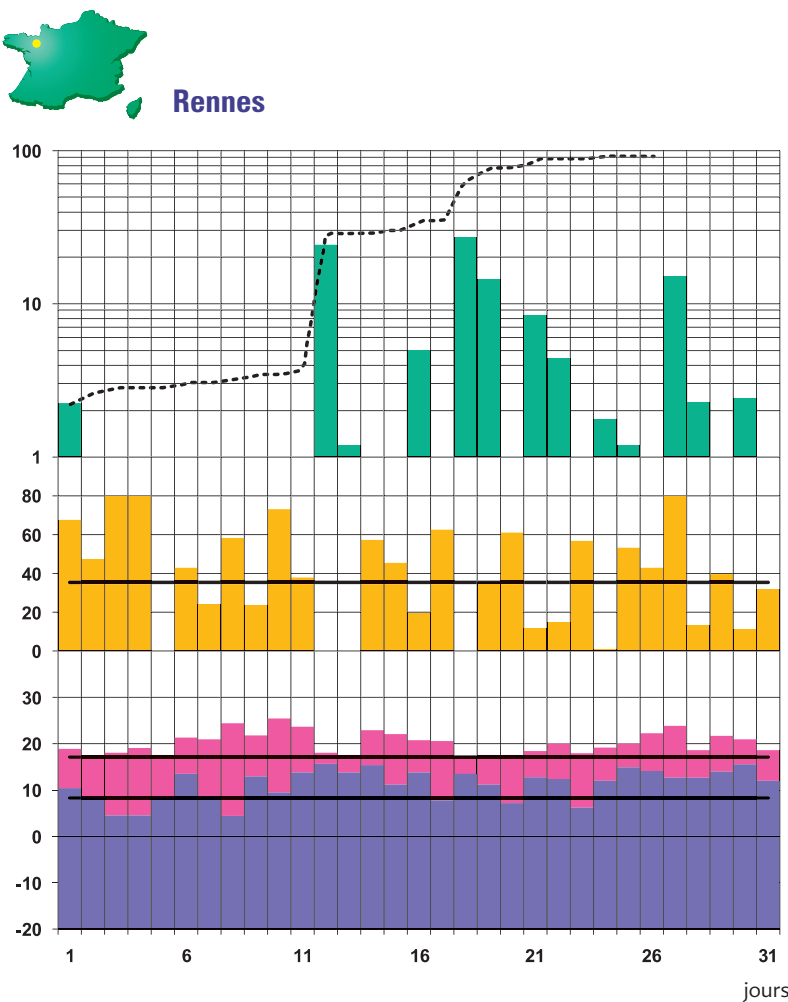

Total mensuel des précipitations : 1,7 fois la normale

Fraction d'insolation moyenne : normale

Température moyenne : $>3,2^{\circ} \mathrm{C}$ à la normale
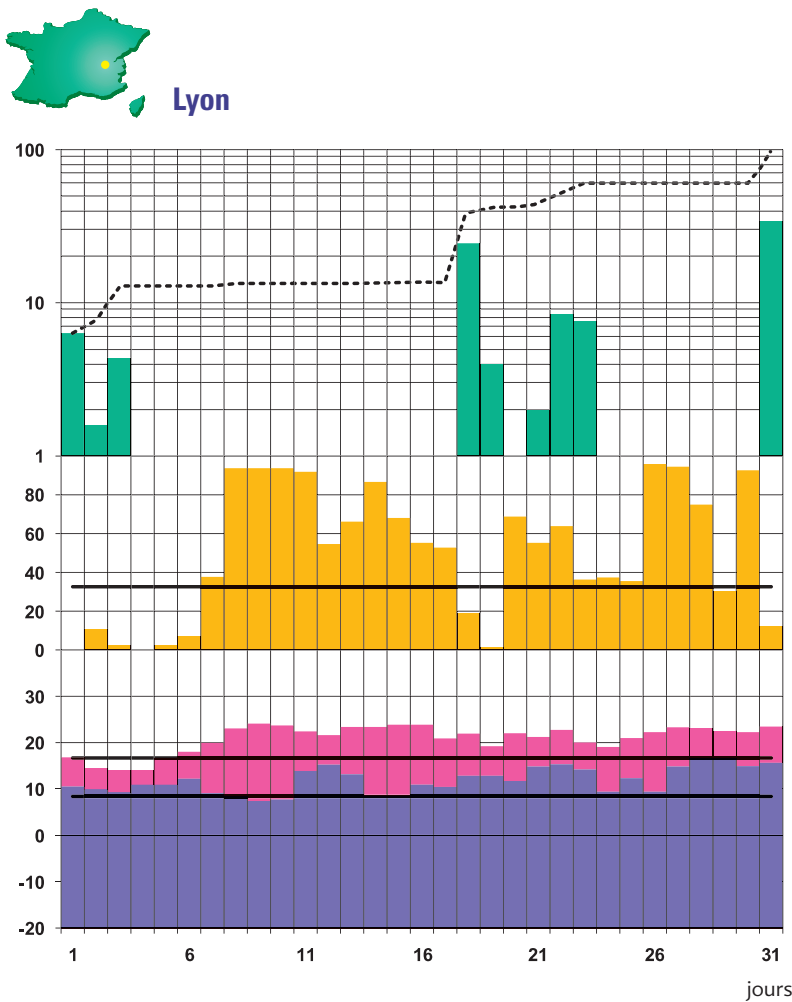

Total mensuel des précipitations : normal

Fraction d'insolation moyenne : excédentaire

Température moyenne : $>3,9^{\circ} \mathrm{C}$ à la normale

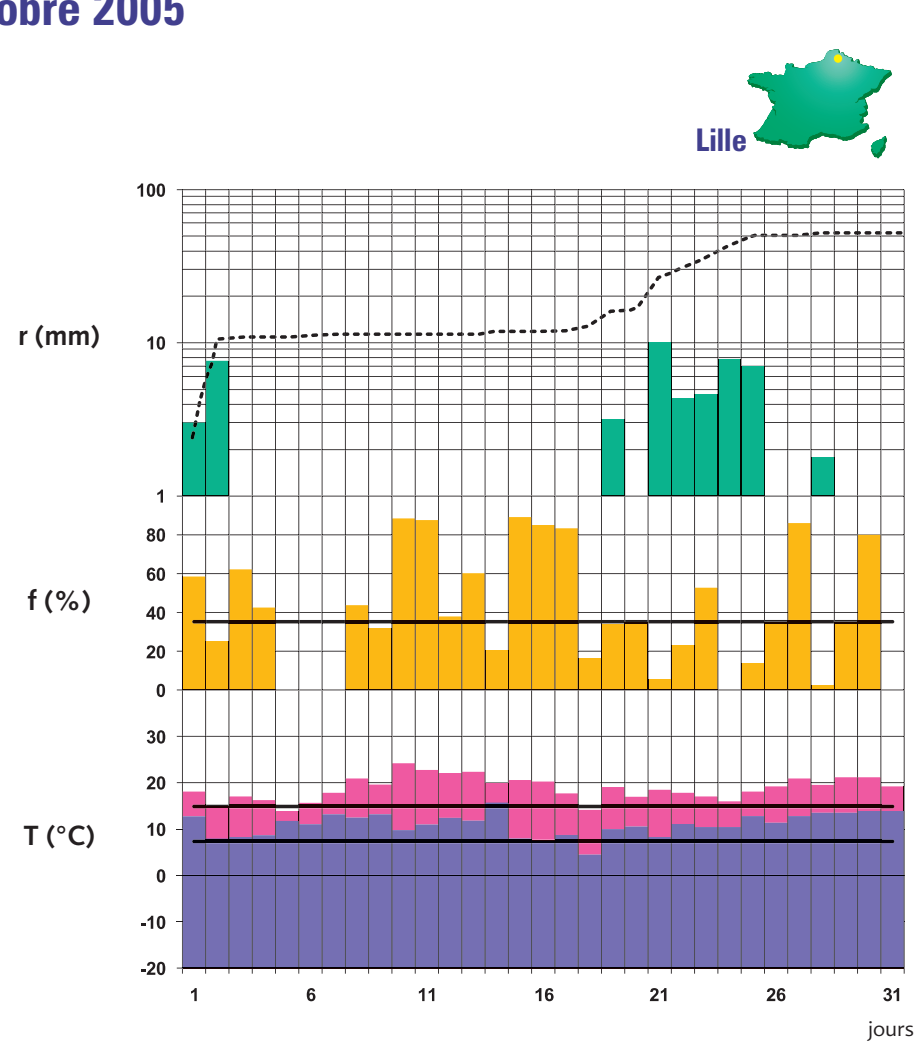

Total mensuel des précipitations : $80 \%$ de la normale

Fraction d'insolation moyenne : légèrement excédentaire

Température moyenne : $>3,8^{\circ} \mathrm{C}$ à la normale

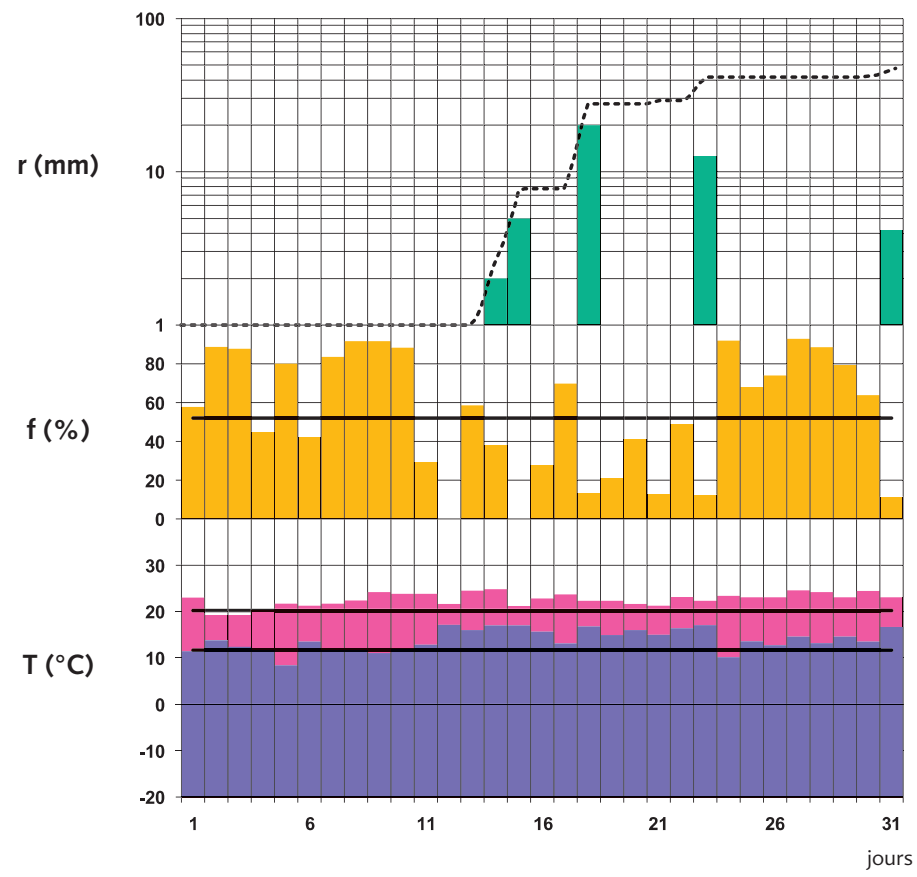

Total mensuel des précipitations : $50 \%$ de la normale

Fraction d'insolation moyenne : normale

Température moyenne : $>2,3{ }^{\circ} \mathrm{C}$ à la normale

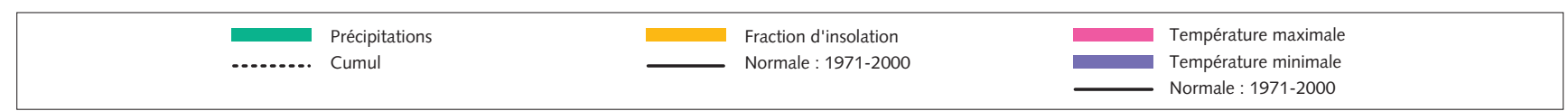




\section{Octobre 2005}
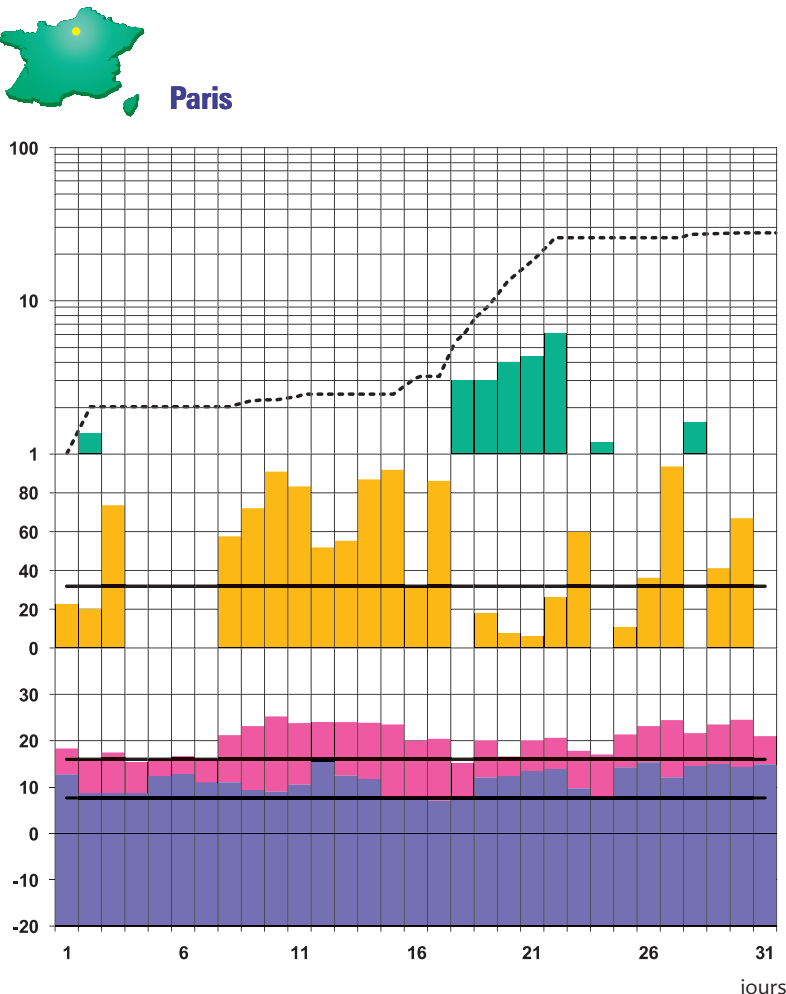

Total mensuel des précipitations : $40 \%$ de la normale

Fraction d'insolation moyenne : excédentaire

Température moyenne : $>4,1^{\circ} \mathrm{C}$ à la normale
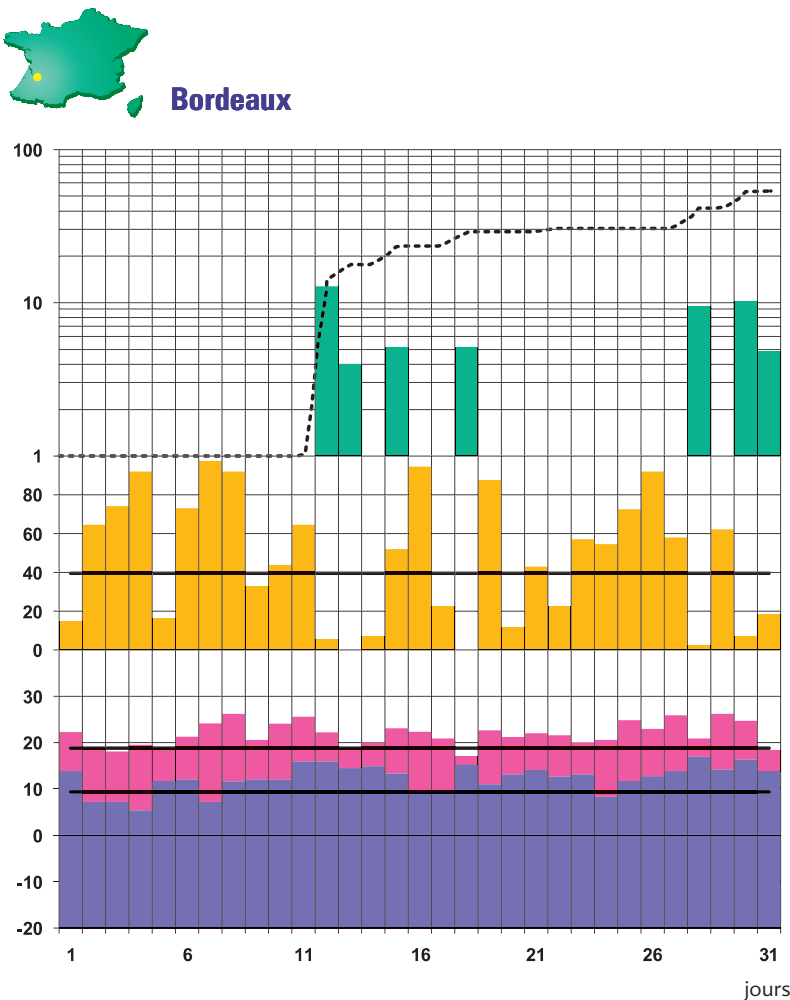

Total mensuel des précipitations : $60 \%$ de la normale

Fraction d'insolation moyenne : légèrement excédentaire

Température moyenne : $>2,9{ }^{\circ} \mathrm{C}$ à la normale

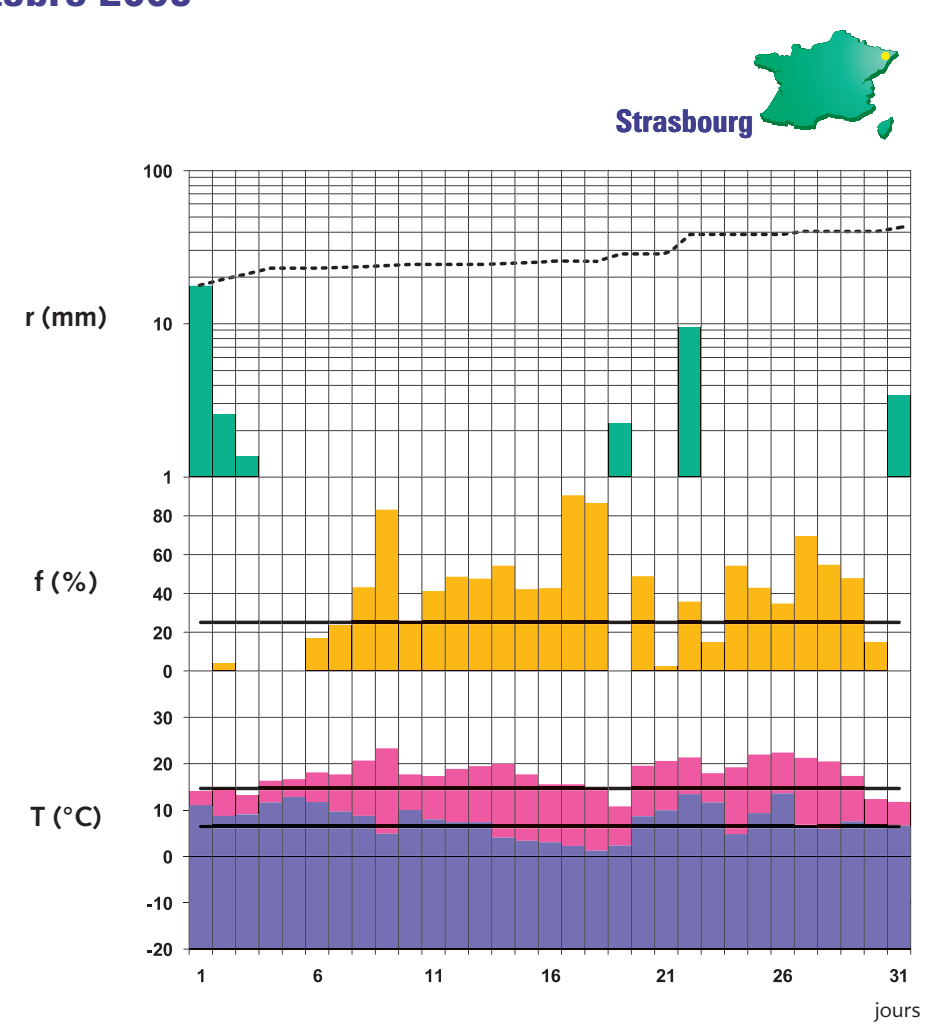

Total mensuel des précipitations : $80 \%$ de la normale

Fraction d'insolation moyenne : excédentaire

Température moyenne : $>2,1^{\circ} \mathrm{C}$ à la normale

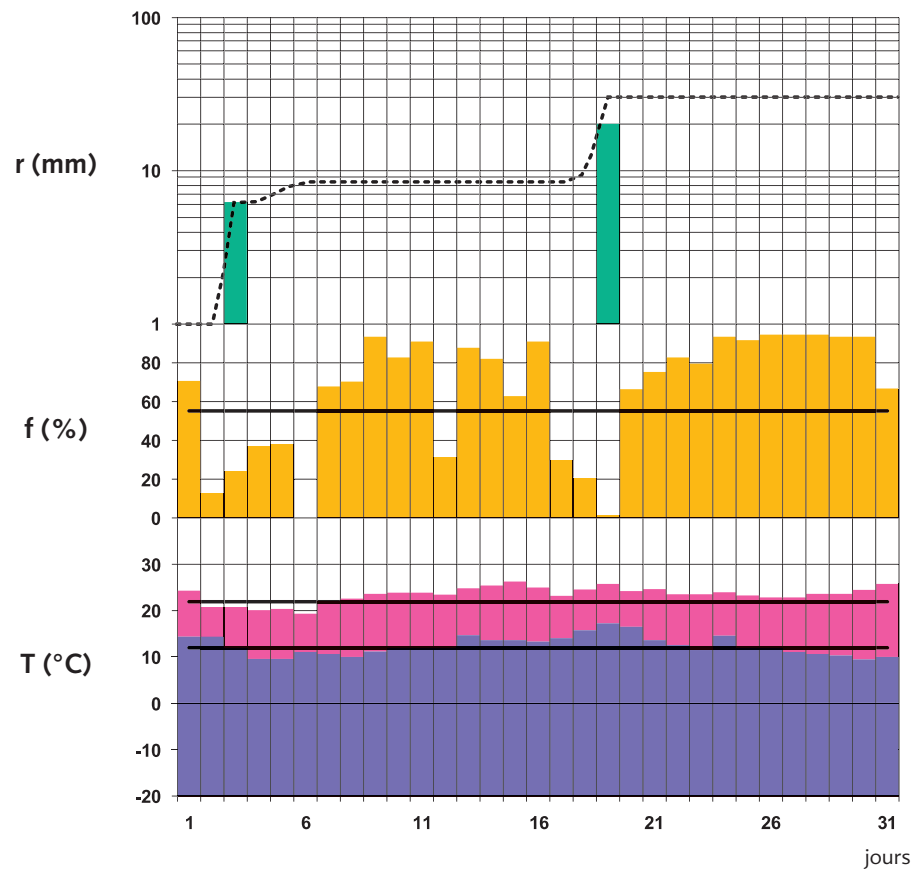

Total mensuel des précipitations : $30 \%$ de la normale

Fraction d'insolation moyenne : légèrement excédentaire

Température moyenne : $>1,1^{\circ} \mathrm{C}$ à la normale

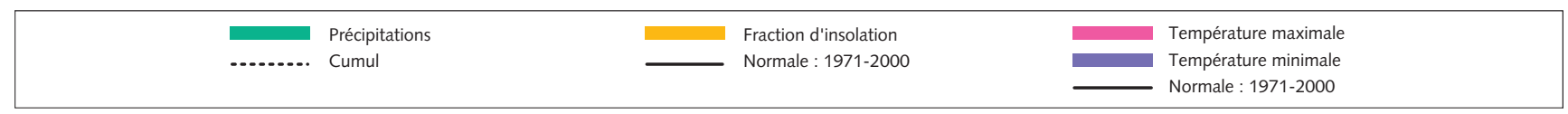


À l'exception de la première décade un peu fraîche sur le sud du territoire, le reste du mois est particulièrement chaud sur la quasitotalité du pays et de nombreux records décadaires sont battus. Avec une anomalie de température de $+3,2^{\circ} \mathrm{C}, 0 \mathrm{octobre} 2005$ se classe au deuxième rang des mois d'octobre les plus chauds depuis 1950, derrière $2001\left(+3,5^{\circ} \mathrm{C}\right)$ et devant $1995\left(+3,1^{\circ} \mathrm{C}\right)$, selon le classement établi à partir de la moyenne de la température moyenne de vingt-deux stations métropolitaines. Les régions île-deFrance, Centre et Bourgogne enregistrent l'anomalie maximale comprise entre $+4^{\circ} \mathrm{C}$ et $+5^{\circ} \mathrm{C}$, alors que la frange sud varie entre $-1^{\circ} \mathrm{C}$ et $+2^{\circ} \mathrm{C}$. Certaines stations enregistrent le mois d'octobre le plus chaud depuis $1950: 16,1^{\circ} \mathrm{C}$ à Paris-Montsouris [deuxième rang : $15,8^{\circ} \mathrm{C}$ en 2001 ], $16,4^{\circ} \mathrm{C}$ au Mans (Sarthe) [deuxième rang : $15,7^{\circ} \mathrm{C}$ en 2001].

Des précipitations soutenues et durables se déversent longuement autour du golfe du Lion. Des pluies significatives concernent également l'ouest du pays, améliorant sensiblement la situation sur ces régions toujours éprouvées par la sécheresse des sols superficiels.

En deuxième et surtout troisième décades, un fort vent de sud - sud-est souffle dans le domaine de l'autan, permettant un excédent important du nombre de jours de vent $\geq 16 \mathrm{~m} / \mathrm{s}(58 \mathrm{~km} / \mathrm{h})$ sur les stations concernées. En revanche, peu de vent sur les rivages, à part le marin.

Les puissantes et persistantes entrées maritimes génèrent un déficit d'ensoleillement très notable autour du golfe du Lion ; à l'inverse, le soleil s'est montré très généreux sur le Nord-Est à partir de la deuxième décade.

\section{Périodes thermiques}

\section{Un mois exceptionnellement chaud}

\section{Première décade}

Minimales. Les petits matins sont audessus des moyennes saisonnières le $1^{\text {er }}$ sur une grande partie du territoire, mais ils perdent quelques degrés les 2 et 3 et restent froids le 4 , principalement sur l'Ouest en raison d'un bon rayonnement nocturne : $13{ }^{\circ} \mathrm{C}$ à Fontainebleau (Seine-et-Marne) [normale $6,4^{\circ} \mathrm{C}$ ] le $1^{e r} ; 3,1^{\circ} \mathrm{C}$ à Jarnac (Charente) [normale $9,2^{\circ} \mathrm{C}$ le $2 ; 1,1^{\circ} \mathrm{C}$ à Condé-surVire (Manche) [normale $8,1^{\circ} \mathrm{C}$ ] le 3 ; $0,8{ }^{\circ} \mathrm{C}$ à Mont-de-Marsan (Landes) [normale 9, $6{ }^{\circ} \mathrm{C}$ ] le 4 .

Puis, du 5 au 8 , la forte nébulosité qui prédomine sur le nord de l'Hexagone permet au thermomètre d'enregistrer des excédents, alors que, dans le Sud, c'est la fraîcheur qui l'emporte : $5,3{ }^{\circ} \mathrm{C}$ à Salon-de-Provence (Bouches-duRhône) [normale $11,4{ }^{\circ} \mathrm{C}$ ] et $13,5^{\circ} \mathrm{C}$ à Condécourt (Val-d'Oise) [normale 7,3 $\left.{ }^{\circ} \mathrm{C}\right]$ le $5 ; 5,1{ }^{\circ} \mathrm{C}$ à Montauban (Tarn-et-Garonne) [normale 10,3 ${ }^{\circ} \mathrm{C}$ ] et $12,8^{\circ} \mathrm{C}$ à Fontainebleau (Seine-etMarne) [normale $6,4{ }^{\circ} \mathrm{C}$ )] le $6 ; 4,9^{\circ} \mathrm{C}$ à Durban (Aude) [normale 11,9 ${ }^{\circ} \mathrm{C}$ ] et $14{ }^{\circ} \mathrm{C}$ à Romorantin (Loir-et-Cher) [normale $7,5{ }^{\circ} \mathrm{C}$ ] le $7 ; 5,1{ }^{\circ} \mathrm{C}$ à Donzère (Drôme) le 8.

$\mathrm{Si}$, le 9, les fins de nuit sont encore agréables pour la saison de l'Anjou à l'Artois, le reste de l'Hexagone - principalement la pointe bretonne et le Sud-Est - enregistre des déficits. Le 10, les minimales sont proches des normales, plutôt chaudes à l'Ouest et froides à l'Est.

Maximales. En ce premier jour d'octobre, les maximales sont généralement de sai- son, avec toutefois quelques excédents notables sur le Midi méditerranéen et un déficit plus marqué des Savoies à 1'Alsace. La fraîcheur est ensuite de mise des Pyrénées au Nord-Est et aux Alpes du 2 au 4, en raison d'un soleil qui brille par son absence : $11,6{ }^{\circ} \mathrm{C} \grave{a}$ Die (Drôme) [normale $18,9^{\circ} \mathrm{C}$ ] le 2 ; 10,5 ${ }^{\circ} \mathrm{C}$ à Arbois (Jura) le $3 ; 10,4{ }^{\circ} \mathrm{C}$ à Belmont-sur-Rance (Aveyron) [normale $17,7^{\circ} \mathrm{C}$ ] le 4 .

Malgré la forte nébulosité qui persiste le 5 , le mercure remonte très légèrement. Cette tendance se confirme jusqu'à la fin de la décade ; ainsi, le 6, les températures reviennent-elles à un niveau de saison, qu'elles dépassent le 7, sauf de la Beauce à la Picardie et sur le pourtour méditerranéen : $17,9^{\circ} \mathrm{C}$ à Alistro (Haute-Corse) [normale $\left.22,4^{\circ} \mathrm{C}\right]$ et $25,3^{\circ} \mathrm{C}$ à Thouars (DeuxSèvres) [normale $18,6^{\circ} \mathrm{C}$ ] le 7.

À partir du 8, la plupart des valeurs dépasse les $20^{\circ} \mathrm{C}$ et des records décadaires sont battus le 10 dans le Nord; le littoral méditerranéen reste à l'écart de cette hausse du mercure : $28^{\circ} \mathrm{C}$ à Bazas (Gironde) [normale $20^{\circ} \mathrm{C}$ ] le 8 ; $24,2^{\circ} \mathrm{C}$ à Aubusson (Creuse) [normale $15,9^{\circ} \mathrm{Cl}$ le $9 ; 18^{\circ} \mathrm{C}$ à Gruissan (Aude) [normale $21,2{ }^{\circ} \mathrm{C}$ ], 24, ${ }^{\circ} \mathrm{C}$ à Argentan (Orne) et $25,6^{\circ} \mathrm{C}$ à Saint-Sylvain (Calvados) le 10.

\section{Deuxième décade}

Minimales. Dans un flux souvent orienté de secteur sud-est, elles sont douces pour la saison sur presque toute la décade, à l'exception du 17, de manière plus importante sur l'ouest du pays. Des records décadaires de chaleur sont dépassés. Le quart nord-est, bénéficiant d'un ciel nocturne souvent dégagé, enregistre des déficits quasi quotidiens : $16,1{ }^{\circ} \mathrm{C}$ à Archigny (Vienne) le 11 ; $17,6{ }^{\circ} \mathrm{C}$ à Bouteville
(Charente) le $12 ; 16,5^{\circ} \mathrm{C}$ au Perrier (Vendée) le 13 ; 15,5 ${ }^{\circ} \mathrm{C}$ à Abbeville (Somme) le $14 ;-0,1{ }^{\circ} \mathrm{C}$ à Épinal (Vosges) [normale $6{ }^{\circ} \mathrm{C}$ ] et $16,8^{\circ} \mathrm{C}$ à Roquevaire (Bouches-du-Rhône) le 15 ; $0,6{ }^{\circ} \mathrm{C}$ à Mulhouse (Haut-Rhin) [normale $6,7^{\circ} \mathrm{C}$ ) et $14^{\circ} \mathrm{C}$ à Bourbonl'Archambault (Allier) le 16.

En fin de nuit du 17, on oscille autour des normales, mais le mercure remonte dès le 18 , sauf sur le quart nord-est : $-1,6{ }^{\circ} \mathrm{C}$ à Colmar-Meyenheim (HautRhin) [normale $6,7^{\circ} \mathrm{C}$ ] et $16,3{ }^{\circ} \mathrm{C}$ à Bonnieux (Vaucluse) [normale $8,8^{\circ} \mathrm{C}$ ] le 18 .

Le 19, l'Alsace et un petit quart sudouest, auxquels se joint la Bretagne le 20, restent sous les normales : $4{ }^{\circ} \mathrm{C} \grave{a}$ Saint-Ségal (Finistère) [normale $\left.8,7{ }^{\circ} \mathrm{C}\right]$ et $14,2{ }^{\circ} \mathrm{C}$ à Saint-Paul-lèsDurance (Bouches-du-Rhône) [normale $7,1^{\circ}$ C] le 20.

Maximales. De forts excédents sont relevés jusqu'au 15 , surtout sur les deux tiers nord du territoire et de nombreux records décadaires sont franchis. Un bémol le 13 où le Nord-Est est le seul à conserver de hautes valeurs. Au sud du $45^{\mathrm{e}}$ parallèle, le ciel souvent chargé ne permet pas au mercure de monter : $27,2{ }^{\circ} \mathrm{C}$ à Lège-Cap-Ferret (Gironde) le $11 ; 14,8^{\circ} \mathrm{C}$ à Conqueyrac (Gard) [normale $\left.20^{\circ} \mathrm{C}\right]$ et $25,4{ }^{\circ} \mathrm{C}$ à Argenteuil (Val-d'Oise) le $12 ; 13,7^{\circ} \mathrm{C}$ à Bédarieux (Hérault) [normale $17,9{ }^{\circ} \mathrm{C}$ ] le 13 ; $24,8{ }^{\circ} \mathrm{C}$ à Hennebont (Morbihan) le 14 ; 23, $6^{\circ} \mathrm{C}$ à Pouilly-en-Auxois (Côted'Or) le 15.

Même si la grande majorité des stations enregistre encore des excédents les 16 et 17 , ces derniers sont globalement moins importants. Les températures baissent encore le 18 et les valeurs oscillent autour des normales. Le 19, les maximales sont normales en Bretagne et en Normandie, chaudes du Béarn au Massif 
central et au Nord - Pas-de-Calais et fraîches sur la frange est : $14,1{ }^{\circ} \mathrm{C} \grave{a}$ Roquesteron (Alpes-Maritimes) [normale 20,1 ${ }^{\circ} \mathrm{C}$ ] et $23,8^{\circ} \mathrm{C}$ à Decize (Nièvre) [normale $17^{\circ} \mathrm{C}$ ] le 19 .

Le lendemain la zone d'air chaud se déplace des Pyrénées à l'Alsace et seul l'extrême Sud-Est enregistre des déficits notables.

\section{Troisième décade}

Minimales. Durant cette vague de grande douceur, seule la nuit du 23 au 24 reste globalement dans la moyenne, à l'exception de la pointe bretonne : $14,7{ }^{\circ} \mathrm{C}$ à Sainte-Enimie (Lozère) le $21 ; 13{ }^{\circ} \mathrm{C}$ à Juzennecourt (HauteMarne) le 22 ; $16,5{ }^{\circ} \mathrm{C}$ à Bonnieux (Vaucluse) le 23.

À partir du 24, les Pyrénées, les Alpes et la Corse passent souvent sous les normales, alors qu'ailleurs, la douceur s'accentue à partir du 26 pour culminer les 28 et $29: 16,3{ }^{\circ} \mathrm{C}$ à la pointe de Penmarch (Finistère) le $24 ; 16,8^{\circ} \mathrm{C}$ à Guérande (Loire-Atlantique) le 25 ; $14,4{ }^{\circ} \mathrm{C}$ à Metz-Frescaty (Moselle) le $26 ; 4,1{ }^{\circ} \mathrm{C}$ à Vic-en-Bigorre (HautesPyrénées) [normale $6,6^{\circ} \mathrm{C}$ ] et $18,7^{\circ} \mathrm{C}$ à Dourgne (Tarn) le $27 ; 19,4{ }^{\circ} \mathrm{C}$ à Dangé-Saint-Romain (Vienne) le 28 ; $18,7{ }^{\circ} \mathrm{C}$ à Charlieu (Loire) le 29 ; $4,4^{\circ} \mathrm{C}$ à Corte (Haute-Corse) [normale $\left.7,5{ }^{\circ} \mathrm{C}\right]$ et $23,3{ }^{\circ} \mathrm{C}$ à Biarritz (Pyrénées-Atlantiques) le $30 ; 19,2{ }^{\circ} \mathrm{C}$ à Torreilles (Pyrénées-Orientales) le 31 .

Maximales. Elles sont très agréables pour la saison les 21 et 22 sur une très grande partie du pays, de manière plus nette du Sud-Ouest au Nord-Est ; les quelques déficits enregistrés restent faibles : 22,6 ${ }^{\circ} \mathrm{C}$ à Rouffach (Haut-Rhin) [normale $13{ }^{\circ} \mathrm{C}$ le $21 ; 16,2^{\circ} \mathrm{C}$ à Bargemon (Var) [normale $20,3{ }^{\circ} \mathrm{C}$ ] et $22,4^{\circ} \mathrm{C}$ à Strasbourg (Bas-Rhin) [normale $13{ }^{\circ} \mathrm{C}$ ] le 22.

Le mercure baisse sensiblement le 23, même si le plus grand nombre de stations reste au-dessus des moyennes saisonnières. Il remonte dès le 24 et les premiers records tombent dès le 26 et ce jusqu'au 31, même si ce dernier jour, en raison d'une importante nébulosité, les forts excédents ne se cantonnent plus qu'à un très grand quart nord-est. Durant cette période, les maximales restent de saison du Languedoc à la Margeride : $24,9{ }^{\circ} \mathrm{C}$ à Chablis (Yonne) [normale $14,6{ }^{\circ} \mathrm{C}$ ] le $25 ; 25,7^{\circ} \mathrm{C}$ à Bessines (Haute-Vienne) le $26 ; 26,7^{\circ} \mathrm{C}$ à Jarnac (Charente) le $27 ; 25,4{ }^{\circ} \mathrm{C}$ à Pouilly-sur-Loire (Nièvre) le 28 ; $24,5{ }^{\circ} \mathrm{C}$ à Saint-Pourçain-sur-Besbre (Allier) le 29 ; $26,7^{\circ} \mathrm{C}$ à Saint-AmandMontrond (Cher) le $30 ; 26,8{ }^{\circ} \mathrm{C}$ à Saint-Jean-en-Royans (Drôme) le 31.

\section{Périodes pluviométriques}

\section{Une deuxième partie de mois très agitée}

\section{Première décade}

La limite qui s'étire le $1^{\text {er }}$ des Pyrénées au Nord-Est se montre plus particulièrement du Morvan à la Savoie et au nord de l'Alsace. À l'arrière, la traîne s'active le long des côtes de la Manche, à proximité de l'air le plus froid : 31,2 mm à Bonneville (Haute-Savoie), 31,4 mm à Busson (Haute-Marne) et 37,5 mm à Chaux-des-Près (Jura).

La perturbation qui a fini d'évacuer le pays en fin de nuit laisse la place le 2 à une traîne des Pyrénées au Nord et aux Alpes, surtout dynamique dans sa partie orientale : 24,8 mm à Bons-en-Chablais (Haute-Savoie), $31 \mathrm{~mm}$ à La Chapelleen-Vercors (Drôme) et 49,5 mm à SaintPierre-de-Chartreuse (Isère).

Un minimum d'altitude stationnaire entre Corse et continent favorise l'instabilité et organise des retours sur la moitié est. Les précipitations, localement sous forme de neige au-dessus de 1500 mètres, sont présentes du Roussillon à l'Alsace et à la Corse le 3. On n'observe que peu d'évolution le 4, avec des retours d'est du Rhin au Berry et une instabilité persistante sur l'extrême Sud-Est et la Corse qui s'enroule autour du minimum : $32,2 \mathrm{~mm}$ à Combovin (Drôme) et $40 \mathrm{~mm}$ à Oletta (Haute-Corse) avec de l'orage à Bastia le 3 ; 18 mm à Comps-sur-Artuby (Var) et 32,9 mm à Trois-Épis (HautRhin) le 4.

Si quelques ondées se produisent encore le 5 sur le Centre et de l'Aude aux Pyrénées-Orientales, la situation reste marquée par l'instabilité qui persiste dans le Sud-Est : 19,2 mm à L'ÎleRousse (Haute-Corse), 30,8 $\mathrm{mm}$ à Mouans-Sartoux (Alpes-Maritimes) et $44 \mathrm{~mm}$ à Aups (Var).

Avec le comblement de la goutte froide méditerranéenne, l'instabilité tend à s'amenuiser, mais quelques averses persistent encore le $6: 12,6 \mathrm{~mm}$ au cap Corse (Haute-Corse) avec de l'orage, 16,6 mm à Figari (Corse-du-Sud) et $51,2 \mathrm{~mm}$ à Ristolas (Hautes-Alpes).

Les premières chutes de neige notables sont relevées sur les montagnes corses au-dessus de 2000 mètres avec 3 à 5 centimètres, en moyenne.

Malgré l'éloignement du minimum vers l'Italie, des retours instables avec des averses parfois orageuses persistent sensiblement sur les mêmes régions le $7: 17,8 \mathrm{~mm}$ à Sartène (Corse-du-Sud) et 19,2 $\mathrm{mm}$ à Laragne-Montéglin (Hautes-Alpes) le 7.
La décade s'achève avec des précipitations de moindre intensité.

Le 8, l'instabilité résiduelle donne encore quelques petites averses en Corse : 8,4 mm à Oletta (Haute-Corse). Ailleurs, une perturbation vient aborder les côtes de la Manche ; très rapidement, son déplacement vers le sud est bloqué et elle se désagrège sur place le 9. En fin de journée du 10, quelques gouttes accompagnent les entrées maritimes en provenance du golfe du Lion.

\section{Deuxième décade}

Des entrées maritimes et des retours pluvio-instables affectent les régions méditerranéennes tout au long de cette deuxième décade, avec cependant un petit répit les 16 et 17. Les entrées maritimes donnent dès le 11 des pluies continues sur le sud du Massif central ; dans le flux maritime de secteur sud-est, les pluies restent marquées le 12 , se renforçant même en soirée : $38,8 \mathrm{~mm}$ aux Aires (Hérault) le $11 ; 52,8 \mathrm{~mm}$ à Labastide-Rouairoux (Tarn) et 58,6 mm aux Aires (Hérault) le 12.

Le 13 voit la formation de retours pluvioinstables avec des intensités de précipitations très importantes, surtout au cours de la nuit du 13 au 14. Après une accalmie en journée, une nouvelle dégradation se produit dans le courant de la nuit suivante : 71,2 mm à Saint-Paul-de-Fenouillet (Pyrénées-Orientales), 144,8 $\mathrm{mm}$ à Arquettes-en-Val (Aude) le 13, record quotidien pour un mois d'octobre [précédent record : $52,6 \mathrm{~mm}$ le 26 octobre $1991]$; 42,2 mm à Cornus (Aveyron) et 67,4 mm à La Quille (Hérault) le 14.

L'activité pluvio-orageuse reprend en cours de journée du 15 et les pluies continuent de remonter vers l'ouest, se faisant même localement plus soutenues que la veille : 47,5 $\mathrm{mm}$ à Rouillac (Charente), 84,6 mm à Villefort (Lozère) et 149,6 mm à La Quille (Hérault) le 15. C'est l'accalmie le 16, avec seulement quelques averses résiduelles. De nouvelles entrées maritimes apportent des pluies dans la nuit du 17 au 18 : 26,4 mm au cap Béar (PyrénéesOrientales) le 17.

Le flux méditerranéen de sud-est se réorganise le 18 ; les fortes précipitations envahissent le quart sud-est jusqu'à la Bresse. Elles se renforcent à partir de la mi-journée : $81,5 \mathrm{~mm}$ à Savoillan (Vaucluse), 129,6 $\mathrm{mm}$ au Perthus (Pyrénées-Orientales), record quotidien pour un mois d'octobre [précédent record: 102,8 $\mathrm{mm}$ le 10 octobre $1994]$ et 150,9 mm aux Aires (Hérault). Dans le régime de sud - sud-est, se produit une alimentation en air chaud sur l'extrême Sud-Est le 19. Les précipitations sont soutenues, notamment en 
raison du soulèvement orographique : $70 \mathrm{~mm}$ à Saint-Tropez (Var) et 76,1 mm à Antibes (Alpes-Maritimes).

Les pluies faiblissent le 20, sous les restes des amas instables sur l'extrême Sud-Est ainsi qu'au sud du Massif central où elles restent néanmoins continues. Ailleurs, les pluies sont plus épisodiques. Encore faiblement pluvieux le 11 de la Bretagne à la Normandie, un front avance le 12 sur la moitié ouest en s'intensifiant : 59,2 $\mathrm{mm}$ à Maxent (Illeet-Vilaine) et 71,1 $\mathrm{mm}$ à Pontorson (Manche) le 12.

Des averses sous l'air froid se produisent le 16 de la Bretagne à la baie de Seine : 25,5 mm à Saint-Cast-le-Guildo (Côtes-d'Armor).

Une perturbation affecte un grand quart nord-ouest le 18. Elle se décale en faiblissant le 19 du Lyonnais au Nord-Est, une traîne active avec des averses localement orageuses se développant sur le NordOuest : 49,2 mm à Louvigné-du-Désert (Ille-et-Vilaine) le $18 ; 21,8 \mathrm{~mm}$ à SaintGemme-la-Plaine (Vendée) le 19.

Le nord du pays subit encore quelques faibles pluies le 20 .

\section{Troisième décade}

Les remontées humides en provenance de Méditerranée persistent le 21 sur le Sud-Est et ont même tendance à se renforcer, notamment sur les contreforts du Massif central : 77,2 $\mathrm{mm}$ à La GrandCombe (Gard), $100 \mathrm{~mm}$ à Étoile-surRhône (Drôme) et 136,4 mm à Berzème
(Ardèche), record quotidien pour un mois d'octobre [précédent record : 107,1 mm le 20 octobre 1999].

Dans le même temps, le corps pluvieux d'une limite s'enfonce sur le nord du pays : $26 \mathrm{~mm}$ à Merdrignac (Côtesd'Armor) et 46,4 $\mathrm{mm}$ à Hennebont (Morbihan).

Entre la traîne active et l'organisation de plusieurs fronts froids, rares sont les régions comme l'extrême Sud-Est à être épargnées par les pluies ou les averses le 22 : 42,5 mm à Gaillac (Tarn) avec de l'orage à Albi, $70 \mathrm{~mm}$ à Mercurol (Drôme) et 91 mm à Aubenas (Ardèche).

Dans 1'Orne, des averses orageuses donnent $14,4 \mathrm{~mm}$ entre $1 \mathrm{~h} 14$ et $13 \mathrm{~h} 42$ à Alençon.

D'importantes précipitations se produisent encore le 23 du Rhône aux Alpes dans la convergence associée à l'air chaud de basses couches : $47 \mathrm{~mm}$ à Vernoux-en-Vivarais (Ardèche) et $66 \mathrm{~mm}$ en une heure à Valréas (Vaucluse). À l'arrière, la traîne donne des averses sur un grand quart sud-ouest : $34 \mathrm{~mm}$ à Biarritz (Pyrénées-Atlantiques) dont $20,2 \mathrm{~mm}$ entre $9 \mathrm{~h} 00$ et $10 \mathrm{~h} 00$.

Toujours le 23, une nouvelle perturbation arrive par les côtes de la Manche. Les pluies continues se font plus marquées le 24, en particulier de la pointe bretonne au Cotentin : 13,6 $\mathrm{mm}$ au Touquet (Pas-de-Calais) le 23 ; 26,2 $\mathrm{mm}$ à Plouray (Morbihan) et $31,8 \mathrm{~mm}$ à Pont-Hébert (Manche) dont 13,2 mm en une heure le 24.
Avec la hausse des champs, l'activité pluvieuse faiblit rapidement le 25 à mesure que le front tente de s'enfoncer sur le pays. Les dernières précipitations liées à la perturbation lentement rejetée au nord du pays se produisent encore le 26 : 11,8 mm à Calais-Marck (Pas-deCalais) le $25 ; 24,5 \mathrm{~mm}$ à Obernai (Bas-Rhin) le 26.

Des entrées maritimes prises dans le flux de sud donnent à nouveau des pluies à proximité du Massif central : 12,2 mm à Saint-Martin-de-Lansuscle (Lozère) le 26.

L'alimentation maritime continue persiste sur ces régions jusqu'à la fin du mois, les cumuls faiblissant quelque peu le 28:32 mm au Vigan (Gard) le 27 ; 59 mm à La Quille (Hérault) le 29, $48,2 \mathrm{~mm}$ le 30 et 75,2 $\mathrm{mm}$ le 31 ; $150 \mathrm{~mm}$ à Cavaillon (Vaucluse) le 31 . Une limite arrive sur la Bretagne dans la soirée du 27. Son activité se renforce en fin de journée du 28 de la Vendée au Nord et ne donne plus que quelques averses résiduelles le $29: 15 \mathrm{~mm}$ à Rennes-Saint-Jacques (Ille-et-Vilaine) le 27 ; 39,3 mm à Martigné-Briand (Maine-et-Loire) le 28.

Une autre perturbation aborde le Finistère le 29, rentre sur le pays le 30 avant d'onduler sur de nombreuses régions le $31: 17,6 \mathrm{~mm}$ à BrestGuipavas (Finistère) le 29 ; 43,8 mm à Bressuire (Vendée) le 30 ; $56,5 \mathrm{~mm}$ à Courpière (Puy-de-Dôme) le 31.

\section{Écart à la normale de la température moyenne} (degrés Celsius)

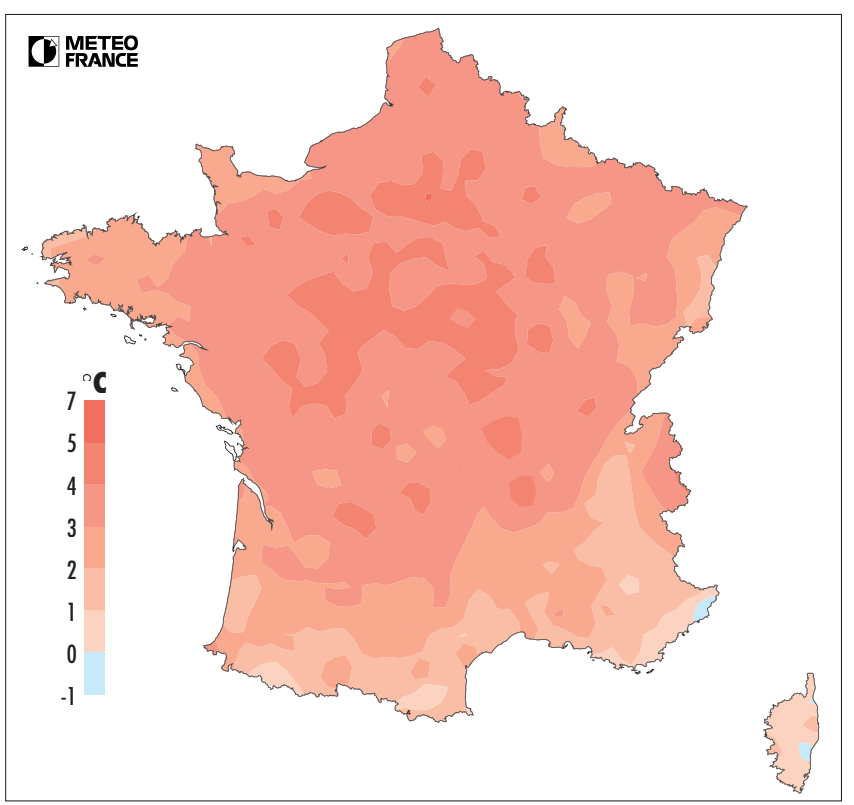

\section{Rapport à la normale des hauteurs de précipitations} (pour-cent)

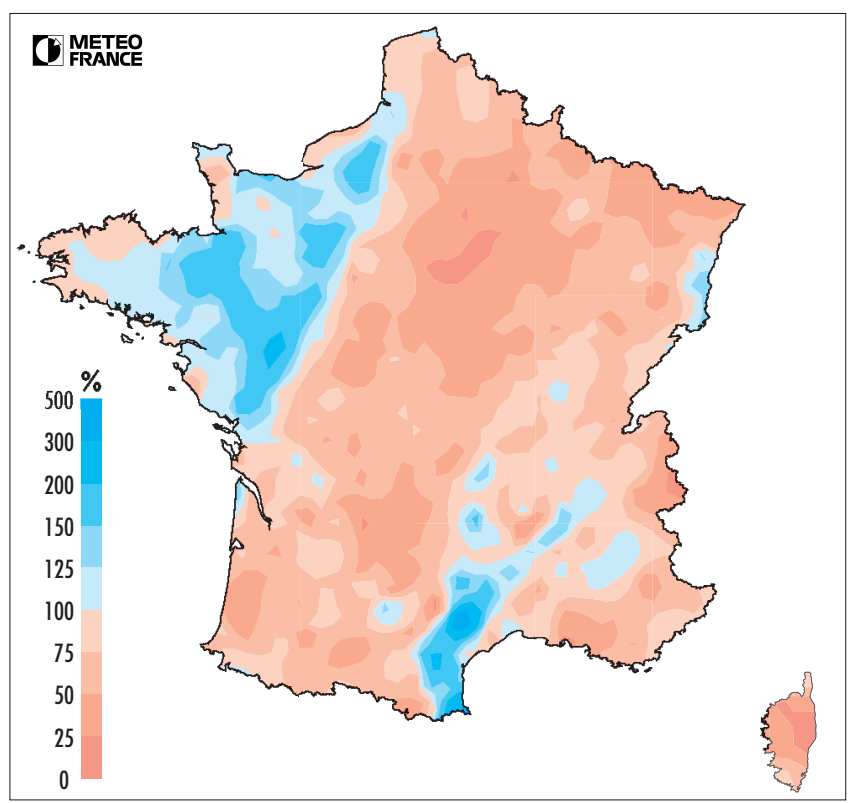

Météo-France DClim 